\title{
Field Evolution of Coexisting Superconducting and Magnetic Orders in $\operatorname{CeCoIn}_{5}$
}

\author{
G. Koutroulakis ${ }^{1}$, M. D. Stewart, Jr. ${ }^{1}$, V. F. Mitrović ${ }^{1}$, M. Horvatić ${ }^{2}$, C. Berthier ${ }^{2}$, G. Lapertot ${ }^{3}$, and J. Flouquet ${ }^{3}$ \\ ${ }^{1}$ Department of Physics, Brown University, Providence, RI 02912, U.S.A. \\ ${ }^{2}$ Laboratoire National des Champs Magnétiques Intenses, \\ CNRS, B.P. 166, 38042 Grenoble Cedex 9, France \\ ${ }^{3}$ CEA, INAC, SPSMS, CEA Grenoble, 38054 Grenoble Cedex 9, France
}

(Dated: October 18, 2018)

\begin{abstract}
We present nuclear magnetic resonance (NMR) measurements on the three distinct In sites of $\mathrm{CeCoIn}_{5}$ with magnetic field applied in the [100] direction. We identify the microscopic nature of the long range magnetic order (LRO) stabilized at low temperatures in fields above $10.2 \mathrm{~T}$ while still in the superconducting (SC) state. We infer that the ordered moment is oriented along the $\hat{c}$-axis and map its field evolution. The study of the field dependence of the NMR shift for the different In sites indicates that the LRO likely coexists with a modulated SC phase, possibly that predicted by Fulde, Ferrell, Larkin, and Ovchinnikov. Furthermore, we discern a field region dominated by strong spin fluctuations where static LRO is absent and propose a revised phase diagram.
\end{abstract}

The detrimental effect of an applied magnetic field on a superconductor has nourished the intuitive presumption of a de facto competition between magnetic and superconducting (SC) orders. However, it is now wellestablished, both theoretically and experimentally, that not only can these orders coexist, but in some cases, they may even be essential for each other's stability [1]. Manifestations of coexistence span a rather wide range of materials including several cuprates, ferropnictides, and heavy fermion systems of which $\mathrm{CeCoIn}_{5}$ is one of the most intriguing examples [2]. In the SC state of this compound application of a magnetic field $\left(H_{0}\right)$ induces a long range magnetic order (LRO), restricted to a narrow lowtemperature $(T)$ region of the phase diagram below $H_{c 2}$ [3, 4]. What is more, this particular region of the phase diagram was initially identified as the first realization of the long-sought Fulde, Ferrell, Larkin, and Ovchinnikov (FFLO) state, a superconducting state with a non-zero pair momentum and a spatially modulated order parameter [5, 6]. However, important questions regarding the true nature of the low- $T$ high- $H_{0}$ SC phase, the details of the magnetic order and its field dependence, and the potential driving mechanisms of their coexistence remain unanswered [7]. Thus, $\mathrm{CeCoIn}_{5}$ provides a strikingly rich ground to study the complex interplay between exotic SC and magnetism. Experimentally, nuclear magnetic resonance (NMR), as a microscopic probe sensitive to both magnetic and SC degrees of freedom, provides a powerful tool for the investigation of these puzzles.

In this letter, detailed low temperature NMR measurements on the three distinct In sites in $\mathrm{CeCoIn}_{5}$ for $\mathbf{H}_{\mathbf{0}} \|$ [100] are presented. We establish that at $T \approx 70 \mathrm{mK}$ a phase with static magnetic LRO is stabilized for fields above $\approx 10.2 \mathrm{~T}$ in the SC state. We deduce that the LRO is an incommensurate spin density wave (IC-SDW) with moments oriented along the $\hat{c}$-axis, independent of the in-plane $\mathbf{H}_{0}$ orientation. Further, the detailed field evolution of the moment is mapped. The study of the field dependence of the NMR shift implies that this IC-SDW coexists with a novel SC state, characterized by an enhanced spin susceptibility [8]. Finally, we identify a new region in the $H_{0}-T$ phase diagram, lying in between the low field SC (lfSC) and the IC-SDW states. This could be an FFLO phase without magnetic LRO, in agreement with recent theoretical predictions [9].

High quality single crystals of $\mathrm{CeCoIn}_{5}$, grown by a flux method, were placed in NMR radio frequency (RF) coils inside the mixing chamber of a dilution refrigerator so that $\mathbf{H}_{\mathbf{0}} \|[100]$. The RF coil was used to determine the precise value of $H_{0}$ by performing ${ }^{63} \mathrm{Cu}$ NMR on its copper nuclei. The spectra were obtained, at each given value of $H_{0}$, from the sum of spin-echo Fourier transforms recorded at constant frequency intervals. Extremely weak RF excitation power [10] was used to discern the NMR signal from In sites sensitive to magnetism.

For $\mathbf{H}_{\mathbf{0}} \|$ [100], there are three inequivalent In sites. The axially symmetric $\operatorname{In}(1)$ is located in the center of the tetragonal Ce planes, while $\operatorname{In}\left(2_{a c}\right)$ and $\operatorname{In}\left(2_{b c}\right)$ sites correspond to In atoms located on the lateral faces (parallel and perpendicular to the applied field, respectively) of the unit cell 11]. In Fig. 1 the $H_{0}$ evolution of the $\operatorname{In}\left(2_{a c}\right)$ and $\operatorname{In}\left(2_{b c}\right)$ spectra at $T \sim 70 \mathrm{mK}$ is plotted. Lowering the field below $\sim 11.7 \mathrm{~T}$ establishes magnetic LRO. The LRO is evident in the fact that the $\operatorname{In}\left(2_{a c}\right)$ line broadens into a spectrum with two extrema/peaks with finite signal weight in between them. Such spectra are characteristic of IC LRO along one spatial dimension [12]. Furthermore, at $H_{0}=11.67 \mathrm{~T}$, both the broad $\operatorname{In}\left(2_{a c}\right)$ and the sharp normal state spectra are observed. This reflects the coexistence of normal and IC phases in the vicinity of the phase transition confirming its first order character. From independent NMR measurements 13] and the tuning resonance of the tank circuit, we establish that this transition to the IC LRO state coincides precisely with the transition from the normal to the SC state. For $9.2 \mathrm{~T} \lesssim H_{0} \lesssim 10.2 \mathrm{~T}$, the spectra of all In sites consist of a single peak, i.e. no signature of the IC state is observed. However, these spectra remain significantly broader than 

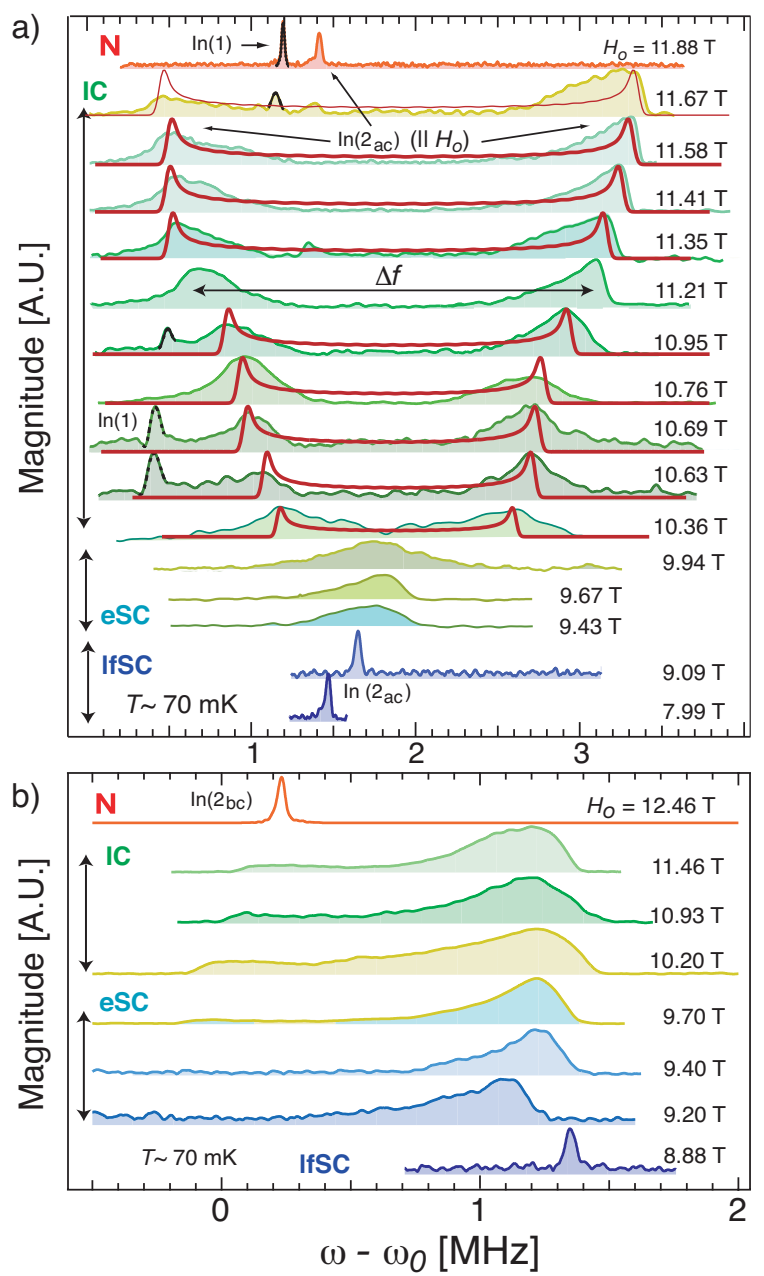

FIG. 1: (Color online) NMR spectra of (a) $\operatorname{In}(1), \operatorname{In}\left(2_{a c}\right)$ and (b) $\operatorname{In}\left(2_{b c}\right)$ at $T=70 \mathrm{mK}$ for various $\mathbf{H}_{\mathbf{0}} \| \hat{a}$. The frequency scale is defined by subtracting $\omega_{0}$, the zero NMR shift frequency. $\mathbf{N}$ denotes the normal phase, IC the LRO phase, eSC the state with strong fluctuations, and lfSC the Abrikosov SC state. Solid lines in (a) are simulated spectra for the IC-SDW order described in the text.

the ones for $H_{0} \lesssim 9.2 \mathrm{~T}$, where the linewidth of all sites can be adequately described by the spatial distribution of magnetic fields resulting from the vortex lattice [13].

We now proceed to the analysis of the nature of the IC LRO phase to elucidate the field evolution of its magnetic moment and its ordering wave vector $(\mathbf{Q})$. The NMR spectrum reflects $\mathcal{P}\left(H_{\text {int }}^{\|}\right)$, the probability distribution of the internal magnetic field at the nuclear site projected along the $\mathbf{H}_{0}$ direction, denoted by $\hat{z}$. In the LRO phase, the component of the internal hyperfine field parallel to $\mathbf{H}_{0}$, at an In site, is given by $H_{\text {int }}^{\|}=\hat{z} \cdot \sum_{\langle i\rangle} \mathbb{A}_{i} \cdot \boldsymbol{\mu}_{i}$, where $\mathbb{A}_{i}$ is the symmetric $3 \times 3$ hyperfine coupling tensor with the $\mathrm{i}^{\text {th }}$ nearest-neighbor Ce atom and $\boldsymbol{\mu}_{i}$ is its magnetic moment [14]. The exact form of the hyperfine tensor is derived in Ref. [15]. Our data and the fact that certain off-diagonal elements of $\mathbb{A}\left(A_{a b}, A_{a c}\right.$, and $A_{b c}$ for $\operatorname{In}(1), \operatorname{In}\left(2_{a c}\right)$, and $\operatorname{In}\left(2_{b c}\right)$, respectively) are non-zero allows us to place stringent constraints on the possible nature of the magnetic LRO. Specifically, the form of $\mathbf{Q}$ and $\boldsymbol{\mu}_{i}$ should be such that the $\operatorname{In}\left(2_{a c}\right)$ spectrum (Fig. 1a) broadens into a double peak structure, while no such broadening of the $\operatorname{In}(1)$ [13] and $\operatorname{In}\left(2_{b c}\right)$ (Fig. 1b) lines is induced. In principle, such spectra can be effectively described by an appropriate sinusoidal variation of $H_{i n t}^{\|}$, that is, of the moment itself. For an ICSDW state, this spatial variation of the moment can be written as $\boldsymbol{\mu}_{i}(\mathbf{r})=\boldsymbol{\mu}_{0} \cos \left(\mathbf{Q} \cdot \mathbf{r}+\phi_{0}\right)$ where $\mathbf{Q}$ is the IC wave vector, $\mathbf{r}$ defines the lattice coordinates of the $\mathrm{i}^{\text {th }}$ Ce moment, and $\phi_{0}$ is an arbitrary phase. The separation between the two extrema of the $\operatorname{In}\left(2_{a c}\right)$ spectrum is $\Delta f=\gamma\left(H_{\text {int }}^{\| \max }-H_{\text {int }}^{\| \min }\right)$, where $\gamma$ is the nuclear gyromagnetic ratio. Thus, $\Delta f$ depends on a product of hyperfine tensor components, the moment amplitude $\mu_{0}$, and some trigonometric function dictated by $\mathbf{Q}$.

A priori, the magnetic structure with the ordered moments $\boldsymbol{\mu}_{i}\left\|\mathbf{H}_{0}\right\| \hat{a}$ and $\mathbf{Q}=\mathbf{Q}_{\mathrm{AF}}+\boldsymbol{\delta}$, where $\mathbf{Q}_{\mathrm{AF}}=$ $(0.5,0.5,0.5)$ and incommensuration $\boldsymbol{\delta}=(\delta, 0,0)$, can satisfy the requirements imposed by our data, as was suggested in [3]. In this case, $\Delta f$ for the $\operatorname{In}\left(2_{a c}\right)$ peaks is equal to $4 \gamma A_{a a} \mu_{0} \sin (\delta \pi)$, with $A_{a a} \approx A_{\operatorname{In}\left(2_{a c}\right)}^{L T} / 2 \approx$ $-0.6 \mathrm{~T} / \mu_{B}$ [11, 15]. However, reproducing the observed spectra requires either a moment $\mu_{0}$ that is three times larger than that found by neutrons for $\mathbf{H}_{0} \|[1 \overline{1} 0]$ [4] or an excessive value of $\delta \simeq 0.21$. It is thus unlikely that such a structure with $\boldsymbol{\mu}_{i} \| \hat{a}$ is stabilized in the IC phase.

A better candidate for describing our data is an ICSDW with ordered moments perpendicular to the plane, i.e. $\boldsymbol{\mu}_{i} \| \hat{c} \perp \mathbf{H}_{\mathbf{0}}$ as in [4], and $\boldsymbol{\delta}$ in the plane. We cannot uniquely determine the direction of $\boldsymbol{\delta}$, since the relevant $\mathcal{P}\left(H_{\text {int }}^{\|}\right)$is nearly the same for $\boldsymbol{\delta}\|\hat{a}, \boldsymbol{\delta}\| \hat{b}$, or $\boldsymbol{\delta} \|[110]$. However, the case of $\boldsymbol{\delta} \| \hat{b} \perp \mathbf{H}_{0}$ appears to be the most plausible one, since it would agree with both the experimental

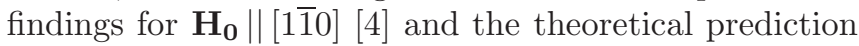
of a $\boldsymbol{\delta} \perp \mathbf{H}_{0}$ for coexisting IC-SDW and FFLO order parameters [9]. In this case, $\Delta f$ is proportional to $4 \gamma A_{a c} \mu_{0}$, where $A_{a c}$ is the off-diagonal hyperfine tensor component, whose value is not known from other independent measurements. Assuming that at $11 \mathrm{~T} \mu_{0}=0.15 \mu_{B}$ and $|\boldsymbol{\delta}|=0.085$ (the same magnitude as in [4]) we find that a reasonable value of $A_{a c} \approx 0.38 \mathrm{~T} / \mu_{B}$ is required to fully account for our data. The calculated distributions $\mathcal{P}\left(H_{\text {int }}^{\|}\right)$, convolved with the underlying vortex lattice lineshape [13], are depicted as the solid lines in Fig. 19. Based on this result, the magnetic LRO for $\mathbf{H}_{0} \| \hat{a}$ is most likely an IC-SDW with the ordered moment perpendicular to the plane. Hence, in conjunction with the neutron findings [4], we infer that the direction of the ordered moments is independent of the in-plane orientation of $H_{0}$.

Having inferred that the magnetic moment is oriented perpendicular to the plane, we proceed to extract the field evolution of its magnitude $\mu_{0}$ from $\Delta f$ of the $\operatorname{In}\left(2_{a c}\right)$ depicted in Fig. 1a. To do so, we fit the data to $\mathcal{P}\left(H_{\text {int }}^{\|}\right)$with $\mu_{0}$ as a fitting parameter and assuming that 


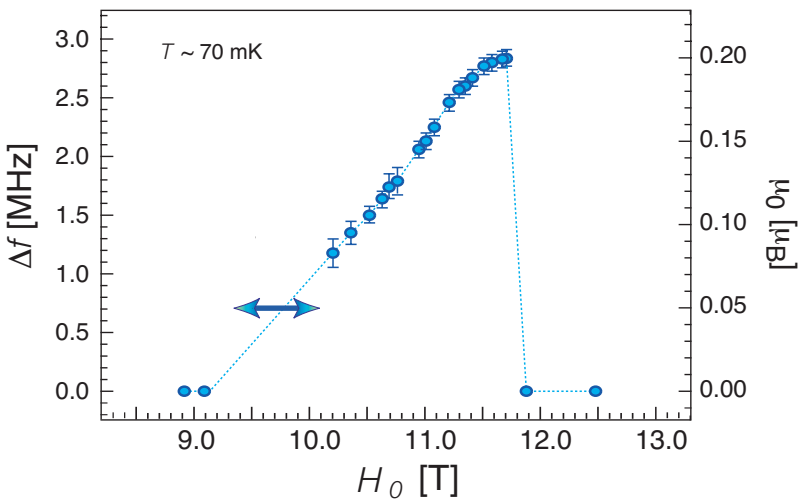

FIG. 2: (Color online) Magnetic moment as a function of $H_{0}$ deduced from $\Delta f$ at $T \approx 70 \mathrm{mK}$. The double arrow denotes the region of $H_{0}$ within which LRO is absent but spectra remain much broader than in the lfSC state.

$|\boldsymbol{\delta}|=0.085$ is $H_{0}$ independent, as experimentally found [4] and theoretically predicted [9, 16], and that the hyperfine component $A_{a c} \approx 0.38 \mathrm{~T} / \mu_{B}$. The deduced field evolution of $\mu_{0}$ is shown in Fig. 2. The moment is zero outside the IC phase below $H_{0} \approx 10.2 \mathrm{~T}$. It increases by a factor of $\sim 2.5$ as the field changes from $\approx 10.3 \mathrm{~T}$ to $11.67 \mathrm{~T}$, where it reaches its maximum value of $\approx 0.2 \mu_{B}$.

In order to better understand the perplexing relationship between the coexisting SC and IC-SDW orders, we next discuss the field dependence of the NMR shift of $\operatorname{In}\left(2_{a c}\right)$ and compare it to that of $\operatorname{In}(1)$ [13]. This allows us to distinguish between the contribution from the low energy local density of states (LDOS), characteristic of the SC state, which affects both sites and that from the localized magnetic moments, affecting predominantly $\operatorname{In}\left(2_{a c}\right)$. Our previous analysis of the $\operatorname{In}(1)$ shift has revealed that a novel SC state, possibly an FFLO state, is stabilized for $H_{0}=H^{*} \gtrsim 10 \mathrm{~T}$ via a $2^{\text {nd }}$ order phase transition [13]. We were, however, unable to exclude the existence of a magnetic order in this novel SC phase. In Fig. 3, the field dependence of the shift, determined by diagonalizing the full nuclear spin Hamiltonian, is plotted. In the lfSC phase, the DOS increases with $H_{0}$ due to excess Zeeman and Doppler-shifted nodal quasiparticles [13], leading to an increasing spin susceptibility $\left(\chi_{s}\right)$. The $\operatorname{In}(1)$ and $\operatorname{In}\left(2_{a c}\right)$ shifts $\left(K \propto A \chi_{s}\right)$ exhibit reversed $H_{0}$ evolution. That is, they scale with their respective hyperfine coupling constants, which are nearly equal but have opposite signs [11], with that for $\operatorname{In}\left(2_{a c}\right)$ being negative. The same is also true for the relative shift change between the normal and lfSC state for both sites.

For $H^{*} \leq H_{0} \leq H_{c 2}$, where the IC-SDW LRO is established, we plot the $\operatorname{In}\left(2_{a c}\right)$ shift of its first moment and of the two extrema of its broadened lineshape. The difference of the shift of the two extrema strongly varies with the field reflecting the increase of $\Delta f$ with increasing $H_{0}$. Further, the $\operatorname{In}\left(2_{a c}\right)$ shift associated with its first moment has essentially the same field dependence as that reported for $\operatorname{In}(1)$, once the difference in their hyperfine

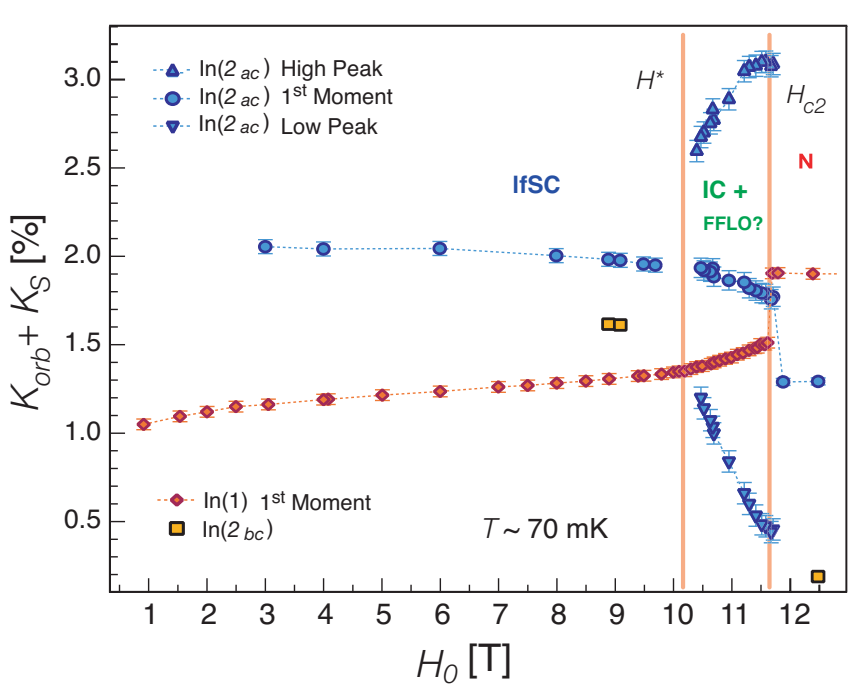

FIG. 3: (Color online) NMR shift (spin, $K_{s}$, and constant orbital part, $K_{\text {orb }}$ [11]) of $\operatorname{In}(1), \operatorname{In}\left(2_{a c}\right)$ and $\operatorname{In}\left(2_{b c}\right)$ at $T \approx 70 \mathrm{mK}$ as a function of $\mathbf{H}_{\mathbf{0}} \| \hat{a}$. The solid lines indicate the values of $H^{*}$ and $H_{c 2}$.

couplings is taken into account. Thus, independently of the local magnetism, both In sites sense an additional common local field, due to a spin polarization along $\mathbf{H}_{0}$.

There are two likely origins for this local field for $H_{0} \geq H^{*}$. One is a canting of the transverse staggered magnetization of the localized moments along $\mathbf{H}_{0}$. To test this hypothesis one needs a quantitative description of canting in an IC phase, which is missing. Thus, we calculate the effect of several crude models of canting on the NMR observables. We find that our data are effectively reproduced by considering an appropriate fixed value of $\mu_{\hat{z}}\left(\sim \mu_{0} \sin \left(5^{\circ}\right)\right)$ on all lattice sites, regardless of $\left|\boldsymbol{\mu}_{i}(\mathbf{r})\right|$. Nevertheless, it is not clear how this type of canted moment could be induced in an IC-SDW phase.

Alternatively, the additional common field could originate from the enhanced LDOS of the spin polarized quasiparticles in the nodal planes of the FFLO state [8]. In that case, since in an FFLO state LDOS varies on a length scale that significantly exceeds the spacing between two In sites, its contribution to the shift of the two distinct In sites should be equivalent as observed. This spatial modulation of the LDOS can also contribute to asymmetric spectral broadening, which for different In sites should scale as their respective hyperfine couplings. For $\operatorname{In}(1)$, this LDOS modulation gives rise to a tail on the high frequency side of the line [10, 13], while for $\operatorname{In}\left(2_{a c}\right)$ the tail should be on the low frequency side, due to the sign difference of their hyperfine coupling. This is indeed observed for $\operatorname{In}\left(2_{a c}\right)$ spectra, where only the low frequency side is essentially broadened beyond IC-SDW lineshape as shown in Fig. 17.

Next, we consider the intriguing low field limit of the IC LRO phase. The low- $T$ shift data provide evidence of a continuous phase transition at $\approx 10.2 \mathrm{~T}$, as shown in Fig. 3. However, significant broadening of $\operatorname{In}\left(2_{a c}\right)$ and $\operatorname{In}\left(2_{b c}\right)$ spectra, as compared to the ones in the lfSC 


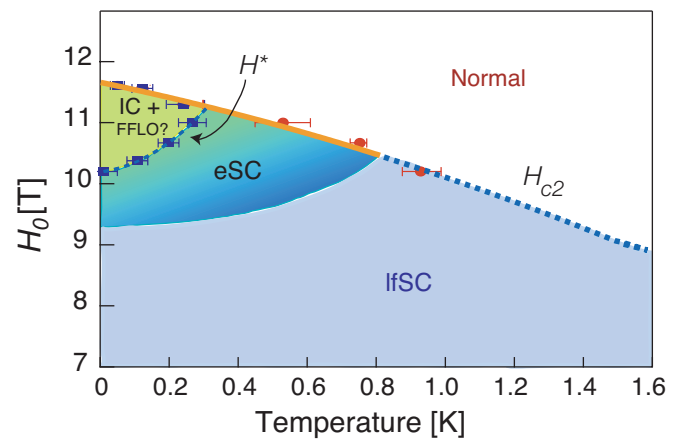

FIG. 4: (Color online) A sketch of an alternative phase diagram of $\mathrm{CeCoIn}_{5}$ (data points adopted from Ref. [10]). The solid and dashed lines indicate $1^{\text {st }}$ and $2^{\text {nd }}$ order phase transitions, respectively.

phase, onsets for $H_{0} \gtrsim 9.2 \mathrm{~T}$, as evident in Fig. 1. Strikingly, $9.2 \mathrm{~T}$ is precisely the field at which the amplitude of the IC-SDW should vanish if one extrapolates from its field dependence in Fig. 2. For $9.2 \mathrm{~T} \lesssim H_{0} \lesssim 10.2 \mathrm{~T}$, the $\operatorname{In}\left(2_{a c}\right)$ signal is weak due to the rapid loss of spin coherence, caused by strong field fluctuations. Thus, although the static LRO is absent, strong antiferromagnetic (AF) fluctuations are still present. These fluctuations can be responsible for the apparent collapse, evident in the disappearance of the double-horned broad $\operatorname{In}\left(2_{a c}\right)$ line, of the LRO in this field region. Besides, the observed additional static broadening, as compared to the lfSC state, in the presence of these fluctuations, can only emerge from highly enhanced $\chi_{s}$ around defects [17], such as nodal planes in an FFLO state. Thus, this field range possibly corresponds to an FFLO state in the presence of strong AF fluctuations, as predicted in [9]. This is all more likely since the width of the low- $T$ spectra of all three In sites in the same field range is comparable to that observed in the higher $T\left(T>T^{*}\right)$, high $H_{0}\left(H_{0}>H^{*}\right)$ SC phase outside the limits of the LRO state [3, 10].

The $2^{\text {nd }}$ order phase transition previously identified by the $\operatorname{In}(1)$ shift can be, then, the transition to the IC LRO state. As recently shown, the IC magnetism can arise in the FFLO state in a $d$-wave $\mathrm{SC}$ as a consequence of the formation of Andreev bound states near the zeros of the FFLO order parameter [9]. It is a large LDOS in the bound states that triggers the formation of the IC LRO [18]. At finite $T$ the LRO phase is stabilized only when a sufficient number of the nodal planes containing the bound states is induced by $H_{0}[9,19]$. Since these same states contribute to the shift, it is likely that the transition identified by it is indeed the one to the IC LRO phase. Alternatively, if the visible increase in the $\operatorname{In}(1)$ shift as compared to that in the lfSC phase were due to canting, an evident increase should onset only after LRO is established.

Based on these observations and consistent with the discussion in the previous paragraphs, we postulate that a phase with strong AF fluctuations (referred to as 'exotic' $\mathrm{SC}(\mathrm{eSC}))$ develops for $9.2 \mathrm{~T} \lesssim H_{0} \lesssim 10.2 \mathrm{~T}$ at low- $T$, while the $2^{\text {nd }}$ order phase transition previously reported [6, 13, 20] at $H^{*} \approx 10.2 \mathrm{~T}$ marks the transition to the state with well established static IC-SDW order. Such a two step phase transition from lfSC to FFLO (assuming it exists in eSC) and then to the IC state was theoretically predicted when spin fluctuations are considered in Ref. [9]. In light of our NMR results, we propose

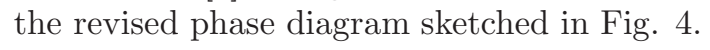

In conclusion, our comprehensive low- $T$ NMR data provide a clear picture of the field evolution of the IC magnetism, and the magnitude of the magnetic moment, confined within the high- $H_{0}$ low- $T$ phase of $\mathrm{CeCoIn}_{5}$. In conjunction with neutron scattering results, we deduce that the direction of the ordered moment does not depend on the in-plane applied field orientation. Our analysis of the field dependence of the NMR shift on different In sites indicates that the IC-SDW order likely coexists with an FFLO state. Finally, we identify a novel phase, in the field regime in between the lfSC and IC-SDW states, which is likely a true FFLO phase in the presence of strong AF fluctuations.

Many thanks to S. Krämer for helping with the experiment. This research is supported by the funds from NSF (DMR-0710551), ANR grant 06-BLAN-0111, and the GHMFL, under European Community contract RITACT-2003-505474. V. F. M. acknowledges support by the A. P. Sloan Foundation.

[1] P. Monthoux et al., Nature 450, 1177 (2007).

[2] J. Flouquet et al., C. R. Physique 7, 22 (2006).

[3] B.-L. Young et al., Phys. Rev. Lett. 98, 036402 (2007).

[4] M. Kenzelmann et al., Science 321, 1652 (2008).

[5] P. Fulde and R.A. Ferrell, Phys. Rev. 135, A550 (1964); A.I. Larkin and Y.N. Ovchinnikov, Zh. Eksp. Teor. Fiz. 47, 1136 (1964).

[6] Y. Matsuda and H. Shimahara, J. Phys. Soc. Jpn. 76, 051005 (2007) (and references therein).

[7] N. J. Curro, Rep. Prog. Phys. 72, 026502 (2009).

[8] V. F. Mitrović et al., Phys. Rev. Lett. 97, 117002 (2006).

[9] Y. Yanase and M. Sigrist, J. Phys. Soc. Jpn. 78, 114715 (2009).

[10] V. F. Mitrović et al., Physica B, 403, 986 (2008).

[11] N. J. Curro et al., Phys. Rev. B 64, 180514 (2001).

[12] R. Blinc, Phys. Rep. 79, 331 (1981).

[13] G. Koutroulakis et al., Phys. Rev. Lett. 101, 047004 (2008).

[14] We assume that itinerant electron contribution is negligible compared to that from the static localized moments.

[15] N. J. Curro, New J. Phys. 8, 173 (2006).

[16] K. Miyake, J. Phys. Soc. Jpn. 77, 123703 (2008).

[17] C. Berthier et al., J. Phys. C 11, 797 (1978).

[18] Y. Yanase and M. Sigrist, J. Phys. 150, 052287 (2009).

[19] A priori, the AF order appears right as the first nodal planes are nucleated at the lfSC-FFLO transition. However, as the dimensionality of the AF order is reduced to the quasi $2 \mathrm{D}$ at very low density of nodal planes, spin fluctuations inhibit LRO at any finite $T$.

[20] A. Bianchi et al., Phys. Rev. Lett. 91, 187004 (2003). 